\title{
Süsse Versuchung, roter Planet oder gar Kriegsgott?
}

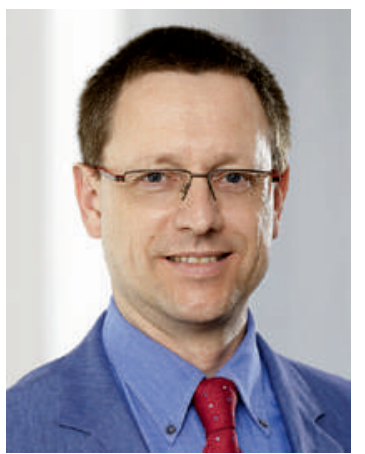

Mars weckt in jedem von uns unwillkürlich verschiedene Assoziationen. Kaum jemand denkt bei diesem Begriff jedoch an ein Bundesprojekt für die Erhebung von Daten zur Gesundheitsversorgung in unserem Land. An der vergangenen Ärztekammer wurde unter Mitwirkung der Verantwortlichen der zuständigen Bundesämter das Projekt «Modules Ambulatoires sur les Relevés de la Sante» (MARS) vorgestellt. In dieser Ausgabe Ihrer Schweizerischen Ärztezeitung werden Sie, liebe Kolleginnen und Kollegen, über dieses Projekt informiert.

Wir finden es unangenehm, tagtäglich mit immer mehr Administration zugemüllt zu werden. Genauso argwöhnisch machen uns Datenerhebungen - dies erstaunt letztlich nicht weiter angesichts dessen, was immer wieder damit angestellt wird und werden kann. Die Politik hat jedoch per Gesetz klare Aufträge erteilt, welche nun ihre Umsetzung finden. Die FMH ist bereits seit längerer Zeit in dieser Thematik aktiv. Es

\section{Der Aufwand soll für das Mitglied, wel- ches seiner gesetzlichen Pflicht nach- kommen will, möglichst gering gehalten werden - die FMH macht das möglich.}

ist uns gelungen, mit den Verantwortlichen des Bundesamtes für Statistik BFS in einen konstruktiven Dialog zu treten. Damit verfolgt die FMH folgendes Anliegen: Durch unsere Zusammenarbeit sollen die Fragen auch aus ärztlicher Sicht qualitativ sinnbildend gestellt werden, um schliesslich auch gute Antworten zu ermöglichen. Für die Ärzteschaft ist nämlich bereits aus dem medizinischen Alltag klar, dass eine gute Indikation bei ordentlich durchgeführter Intervention ein gutes Resultat ergibt. Demgegenüber resultiert aus einer schlechten Indikation auch bei perfekter Intervention kein gutes Resultat. Genauso verhält es sich mit dem Zusammenspiel von Fragen und Antworten.

Des Weiteren soll der Aufwand für das Mitglied, welches seiner gesetzlichen Pflicht zur Datenlieferung nachkommen will, möglichst gering gehalten werden. Die FMH ermöglicht zusammen mit ihren Partnern, dass das Mitglied bereits bestehende ärzteeigene Daten auf Knopfdruck und somit mit seiner Einwilligung einfügen kann. Die datenschutzrechtlichen Aspekte müssen sowohl im Interesse unserer Patienten als auch unserer Mitglieder Beachtung finden - daran arbeiten wir. Hier ist das Bundesamt für Gesundheit BAG gefordert, eine datenschutzkonforme Verordnung vorzulegen.

\section{Gute Fragen sollten aus ärztlicher Sicht qualitativ sinnbildend sein, um schliesslich auch gute Antworten zu ermöglichen.}

Grenzen der Aussagekraft zu erkennen und Doppelspurigkeiten zu vermeiden gilt es, wenn aus statistischen Daten allzu schnell qualitative Rückschlüsse einfach aufgrund einer Koinzidenz gezogen werden. Auch die Grenzen des politischen Auftrags sind zu respektieren: Es kann nicht darum gehen, den im Solidarbereich berechtigten Anspruch des öffentlichen Interesses an Daten aus dem Sozialversicherungsbereich zu missbrauchen, um gleichzeitig auch private Daten zu erheben.

Das BFS stellt in der gleichen Ausgabe der SÄZ das Projekt in einem detaillierten Artikel vor (S. 859). Alle wichtigen Informationen finden Sie ebenfalls auf der Webseite der FMH (www.fmh.ch $\rightarrow$ Politik \& Themen $\rightarrow$ Ärztedemographie $\rightarrow$ MARS).

Mars macht mobil - Ihre FMH wird sich weiterhin konstruktiv und kritisch einsetzen und möchte Ihnen auch bei diesem Thema, sehr verehrtes Mitglied, Unterstützung anbieten.

Dr. med. Christoph Bosshard, Mitglied des Zentralvorstandes der FMH, Departementsverantwortlicher Daten, Demographie und Qualität 